Antonio Sánchez-Padial

\title{
Iniciativas de datos abiertos frente los desafíos de la seguridad alimentaria global
}

INIA (Instituto Nacional de Investigación y Tecnología Agraria y Alimentaria)

Crtra. de la Coruña, km.7,5. 28040, Madrid

\section{Resumen}

Se presenta la relevancia de los datos abiertos para hacer frente al reto global de la seguridad alimentaria mediante una introducción a la iniciativa GODAN (Global Open Data for Agriculture and Nutrition). Se presenta igualmente la participación del INIA en dicha iniciativa, y su proyecto de construcción de un repositorio de datos de investigación agraria. Finalmente se realiza una llamada para unirse a la iniciativa.

\section{Palabras clave}

datos abiertos, seguridad alimentaria, datos de investigación

GODAN es una iniciativa para la promoción de la publicación y utilización de los datos abiertos como herramienta para el desarrollo sostenible, alineada con otras instituciones como OD4D (Open Data for Development).

GODAN nació en octubre de 2013 como resultado de la Conferencia Internacional del G-8 de Datos Abiertos para la Agricultura, celebrada en el mes de abril del mismo año. Sus objetivos, definidos detalladamente en su Statement of Purpose (GODAN, 2013), son promover el intercambio de datos abiertos para hacer que la información sobre agricultura y nutrición esté disponible, sea accesible y utilizable para hacer frente al urgente reto de conseguir la seguridad alimentaria a nivel global.

\section{La participación del INIA en GODAN}

El INIA se sumó a la iniciativa GODAN en diciembre de 2015, como parte de su proyecto de gestión y publicación de datos de investigación. Desde entonces hemos participado en sus talleres, como el celebrado en La Haya en 2015, o la Cumbre GODAN de septiembre de 2016 en Nueva York.

Los datos de investigación son aquellos que son generados o recopilados para el desarrollo de la actividad científica; por su calidad y especialización tienen una especial relevancia para GODAN. Los datos de investigación se incluyen entre las 5 principales categorías de datos relevantes para el desarrollo sostenible de la agricultura, según GODAN, 2016.

La colaboración del INIA con GODAN surge de nuestro interés mutuo en la gestión y publicación de los datos de investigación.

\section{El repositorio de datos de investigación del INIA}

La publicación con licencias abiertas de los datos de investigación es una tendencia creciente en todo el mundo; impulsada por consideraciones éticas y económicas, y promovida tanto por los financiadores de la investigación (H2020, 2013; Gates, 2015) como por los principales editores de revistas científicas (Elsevier, 2015; Nature, 2014) 
A esta necesidad se suma la preocupación por la gestión de los datos de investigación a lo largo de todo su ciclo de vida, con énfasis en su preservación para una posible reutilización futura de los mismos. Esta preservación puede ir o no asociada a su libre acceso, en función de características de los datos como su confidencialidad comercial, la competitividad científica, o la privacidad de los datos de carácter personal.

Con estos objetivos el INIA lanzó el proyecto REDIA (Repositorio de Datos de Investigación Agraria). Este proyecto (García y Sánchez-Padial, 2015), está actualmente en desarrollo y se espera su lanzamiento en el último trimestre de 2017. Con él buscamos garantizar la preservación de los datos generados como parte de la actividad científica de nuestro organismo, así como satisfacer las necesidades de publicación de los mismos: siguiendo los requerimientos de financiadores y editores, y aumentando su difusión y reutilización.

\section{Llamada a la participación}

Como integrante de la iniciativa GODAN, y una vez expuesto el interés de la promoción de los datos abiertos como apoyo para afrontar los retos de la seguridad alimentaria global, os invito a uniros a GODAN y formar parte de los ya casi $500^{1}$ miembros que la integran en todo el mundo.

\section{Bibliografía}

Elsevier, 2015, Elsevier Open Data Pilot. http://www.godan.info/pages/statementpurpose/. (Acceso 16 de marzo de 2017)

Jorge García y Antonio Sánchez-Padial, 2015, Diseño de un repositorio de datos de investigación agroalimentaria. Comunicación no publicada de las XXIV Jornadas Técnicas de RedIRIS. 24 a 26 de noviembre de 2015, Santa Cruz de Tenerife, España. http://doi.org/10.5281/zenodo.232333

Gates, 2014. Bill \& Melinda Gates Foundation Open Access Policy.

http://www.gatesfoundation.org/how-we-work/general-information/open-access-policy. (Acceso 16 de marzo de 2017)

GODAN, 2013, Statement of Purpouse. http://www.godan.info/pages/statementpurposel. (Acceso 16 de marzo de 2017)

GODAN, 2016, Agriculture Open Data Package Working Group. http://www.godan.info/working-groups/agriculture-open-data-package-working-group. (Acceso 16 de marzo de 2017)

H2020, 2013, Piloto para datos de investigación en abierto financiados por Horizonte 2020. http://www.eshorizonte2020.es/actualidad/noticias/piloto-para-datos-deinvestigacion-en-abierto-financiados-por-horizonte-2020. (Acceso 16 de marzo de 2017)

Nature, 2014, Open Research Data Repository Recommendations. Scientific Data. http://www.nature.com/sdata/policies/repositories. (Acceso 16 de marzo de 2017)

\footnotetext{
${ }^{1} 482$ a 7 de marzo de 2017 (http://www.godan.info/partners)
} 